RUNNING HEAD: CHINESE PERIMENSTRUAL SYMPTOMS

\title{
A CULTURALLY SENSITIVE STUDY OF PREMENSTRUAL AND MENSTRUAL SYMPTOMS AMONG CHINESE WOMEN
}

\author{
Antoinette M. Lee, Ph.D. ${ }^{1}$ \\ Catherine So-kum Tang, Ph.D. ${ }^{2}$ \\ Catherine Chong, $\mathrm{MBBS}^{3}$ \\ ${ }^{1}$ Department of Psychiatry, The University of Hong Kong \\ ${ }^{2}$ Department of Psychology, The Chinese University of Hong Kong \\ ${ }^{3}$ Department of Psychiatry, Pamela Youde Nethersole Eastern Hospital, Hong Kong
}

Address for Correspondence and Reprints:

Dr. Antoinette M. Lee

Department of Psychiatry

The University of Hong Kong

2/F, New Clinical Building

Queen Mary Hospital

Pokfulam

Hong Kong

Phone: (852) 28553961 , Fax: (852) 28551345

E-mail: amlee@hkucc.hku.hk 


\begin{abstract}
This is a two-part study of perimenstrual symptomatology in Chinese women. We developed and validated the Chinese Questionnaire of Perimenstrual Symptoms (CQPERI-MS), which was adapted from the Moos Menstrual Distress Questionnaire, and used this instrument to assess the prevalence and nature of perimenstrual symptoms among Chinese women in Hong Kong. The initial CQ-PERI-MS was first administered to a sample of 538 menstruating Chinese women in Hong Kong together with measures of anxiety, depression, and neuroticism. Psychometric analyses rendered a 32-item final CQ-PERI-MS which demonstrated good reliability, convergent and discriminant validity, and factorial validity. Factor analysis yielded four factors, namely, Dysphoria, Somatic Distress, Cognitive Problems, and Arousal. The CQ-PERI-MS was then administered to a separate sample of 339 menstruating Chinese women in Hong Kong for further examination of validity as well as pattern of perimenstrual symptoms. It was found that perimenstrual symptoms were common, with $18.6 \%$ and $34.2 \%$ of the participants reporting ten or more premenstrual and menstrual symptoms respectively. Both premenstrual and menstrual distress were characterized by a combination of emotional and somatic symptoms. Contrary to previous preconceptions, perimenstrual symptoms are commonly experienced by Chinese women, with both overlapping and distinct features as compared to patterns in the West.
\end{abstract}

\title{
Keywords:
}

Premenstrual and Menstrual Symptoms, Premenstrual Syndrome, Chinese Women, Psychometric Properties, Culture

Word count: Abstract (199 words), main text (5986) 


\section{Introduction}

The recurrent nature of menstruation-related changes and its potential impact on the daily lives of women and their significant others makes premenstrual and menstrual symptoms (perimenstrual symptoms, PERI-MS) a central issue in the wellbeing of modern women. While both professional and lay interest in premenstrual syndrome (PMS) has burgeoned in recent years, menstrual distress was largely neglected except for dysmenorrhea. Nevertheless, there is evidence that other symptoms might occur during the menstrual phase, including psychological symptoms such as depressed mood, irritability, and anxiety, and somatic symptoms such as fatigue, nausea, and headache [1-3]. Bancroft also urged for a broader conceptualization of perimenstrual problems that included symptoms in both the premenstrual and menstrual phases [4,5]. Despite increasing awareness of the importance of menstrual complaints, the exact nature of these complaints is not well characterized. Knowledge of PERI-MS among non-Western women is even more lacking, as Johnson [6] and subsequent researchers [7,8] argued that PMS is a Western "culture-bound syndrome". However, there are emerging reports of PMS and its more severe form, Premenstrual Dysphoric Disorder (PMDD), in different non-Western societies including India [9,10], China [11-14], Japan [15], Thailand [16] and Africa [17], suggesting that premenstrual symptoms are also common among non-Western women. Nonetheless, as cultural beliefs are associated with both expectations about menstrual cycles and perimenstrual symptoms [18], perimenstrual symptoms do differ across cultures, in terms of prevalence and symptom patterns [18-22].

Indeed, existing studies showed the pattern of perimenstrual symptoms seem to differ from that depicted among Western women [18-22]. A study of Hong Kong females showed that they were more likely to experience premenstrual fatigue and pain and less likely to experience premenstrual negative affect than Western women [22]. Another study of females in China also showed that premenstrual complaints were predominantly somatic, such as fatigue, hypersomnia, and abdominal pain [23]. More detailed knowledge of the exact nature of premenstrual complaints among Chinese women is, however, not available. Knowledge of Chinese women's experiences during the menstrual phase is even more lacking but one study revealed that over $60 \%$ of Chinese women reported negative physical, psychological, and behavioral changes during the menstrual phase [23], documenting the significance of the problem. Better characterization of both premenstrual and menstrual complaints 
among Chinese women is definitely warranted.

There is a general lack of research on PERI-MS in Chinese women, as menstruation is still a "taboo" in Chinese culture. More importantly, a valid Chinese research instrument for measuring PERI-MS is not available. Previous studies mainly involved very small samples and used instruments derived from the West that were not properly tested for their cultural validity. A previous study translated the widely used Moos Menstrual Distress Questionnaire [MDQ, 24] into Chinese and reported reasonable internal consistency of the Chinese version [22]. However, the issues of factor structure and cultural equivalence, particularly as it related to culturally specific ways of describing premenstrual and menstrual complaints, were not addressed. In other words, the Chinese MDQ demonstrated reliability but questionable validity. Cultural validity requires an instrument to accurately and adequately measure the condition under study in a particular cultural group. As perimenstrual experiences are shaped by cultural beliefs [18], PERI-MS is expected to differ across cultures. The Western-derived MDQ was constructed based on perimenstrual symptoms reported by women living in the West. Moos compiled the list of symptoms in the MDQ through interviewing women in a Western university in USA of their perimenstrual experiences and literature review [24]. Ethnicity of the participants was not reported and there was no indication that it was a culturally diverse group. It is possible that the list of symptoms included in the MDQ may not capture the full range of perimenstrual symptoms experienced by Chinese women, thus underestimating the prevalence of perimenstrual distress of Chinese women. A culturally sensitive instrument that captures the full range of symptoms experienced by Chinese women is needed. As Kleinman [25] urged, culturally sensitive research should go beyond the direct imposition of Western constructs on non-Western contexts in which they might lack validity. In order to derive a locally valid research instrument, complementary "etic" (culture-universal) and "emic" (culture-specific) items should be integrated to form an item pool for validity and reliability analysis. Thus, the development of a culturally sensitive instrument for assessing perimenstrual symptoms among Chinese women should start with an understanding of the perimenstrual experiences as described by Chinese women, through qualitative research approaches such as interviews with Chinese women. It is envisioned that this instrument would include universal symptoms that are experienced by both Western and Chinese women, as 
well as culturally distinct symptoms that are only salient among Chinese women. This instrument also needs to be formally tested for reliability and validity. Without such a culturally valid instrument for measuring PERI-MS among Chinese women, any effort to examine the nature of PERI-MS among Chinese women or to compare PERIMS between Chinese and Western women is dangerous.

Given preliminary evidence of both premenstrual and menstrual complaints among Chinese women, it is of both scientific and clinical relevance to further our understanding of the exact nature of these problems among Chinese women. However, validity of these attempts depends on the availability of a culturally valid instrument for measuring premenstrual and menstrual complaints among Chinese women. Thus, the objectives of the present study are to develop a research instrument that can validly measure PERI-MS among Chinese women, and to use it to examine the prevalence and pattern of PERI-MS among Chinese women in Hong Kong. Results will provide much needed data on perimenstrual experience of Chinese women, and facilitate the development of culturally sensitive research criteria of PERI-MS. In addition, this instrument can, in the future, be used on Western women to examine the universal and culture-specific aspects of PERI-MS.

The study consisted of two parts: Study 1 focused on the development and validation of the Chinese Questionnaire of Perimenstrual Symptoms (CQ-PERI-MS) while Study 2 aimed at confirming the factor structure of the CQ-PERI-MS derived in Study 1 and using the validated CQ-PERI-MS to examine the pattern of perimenstrual symptoms.

\section{STUDY 1}

\section{Methods}

\section{Participants}

The study was approved by the local institutional review board. Informed consent was sought from all participants. A convenience sample of 700 Chinese women in Hong Kong was recruited to participate in the study. Inclusion criteria were ethnic Chinese females between 15 and 55 with regular menstruation. Women who were pregnant, menopausal or amenorrhoeic for other reasons were excluded. A total of 538 women returned the completed questionnaires, yielding a response rate of 
$76.9 \%$.

\section{Development of the CQ-PERI-MS}

The CQPERI-MS was developed from the 47-item Moos Menstrual Distress Questionnaire [MDQ; 24]. The MDQ is a widely used instrument for assessing premenstrual and menstrual emotional, somatic and behavioural changes. In the original MDQ, participants were asked to rate the degree to which they experience each of the 47 symptoms on a six-point scale from 1 (no experience of the symptom) to 6 (acute or partially disabling experience of the symptom), separately for the premenstrual, menstrual, and intermenstrual phases. Premenstrual and menstrual changes can then be derived through subtracting intermenstrual scores from premenstrual and menstrual scores respectively. Eight factors were extracted through factor analysis, representing eight clusters of symptoms which were labelled pain, concentration, behavioural change, autonomic reactions, water retention, negative affect, arousal, and control [24]. The first six factors represented distressing symptoms while arousal referred to positive experiences such as burst of energy and feelings of well-being. The control factor was included to control for tendency to report symptoms regardless of whether they were related to the menstrual cycle, and included symptoms such as "buzzing or ringing in ears" which were not associated with perimenstrual distress.

To complement the list of perimenstrual symptoms characteristic of Western women as depicted in the MDQ, a list of common premenstrual and menstrual symptoms experienced by Chinese women were elicited through semi-structured focus group interviews with menstruating Chinese women conducted by the first author. The aim and format of the interviews were explained to the participants. Confidentiality and anonymity were ensured, and informed consent was obtained. Participants were invited to describe physical, emotional and behavioral changes they experienced in the seven to ten days before menstruation and during the days of menstrual flow. They were encouraged to speak about their experiences freely. Each focus group lasted about 1.5 hours. Consecutive groups of women were interviewed until data saturation was achieved. Data saturation is a well established principle in qualitative research and refers to termination of data collection when information 
from participants becomes repetitive and additional interviews do not contribute significant additional information [26,27]. Based on this principle, three focus group interviews with a total of fourteen Chinese women of diverse age groups and backgrounds were conducted. One focus group included adolescent schoolgirls, another included undergraduate and graduate females, and the final one included working women and housewives. The participants described a mixture of symptoms commonly included in Western instruments as well as symptoms that were rarely assessed in Western instruments, such as "paleness", "body dissatisfaction", and "worrisome". The latter was regarded as locally derived items. A list of 20 locally derived items were complied and added to the 47-tiem MDQ to form the 67-item CQPERI-MS. This enlarged the scope of symptoms surveyed by taking account of the more locally relevant idioms of distress. It was hoped that the resultant CQ-PERI-MS could better capture the full range of perimenstrual symptoms experienced by Chinese women in Hong Kong, and thus be more culturally sensitive.

The psychometric properties of the CQ-PERI-MS, including its factor structure, internal reliability, and convergent and discriminant validity, were examined. Factor structure of the CQ_PERI_MS was examined using exploratory factor analysis. Internal reliability was assessed in terms of internal consistency. In psychometric testing, convergent and discriminant validity is assessed through examination of the relationship between the measure under study (CQ-PERI-MS) and measures of related variables that the construct is expected to have relationship with $[28,29]$. Convergent validity is evidenced when the measure under study is significantly correlated with measures which it theoretically is expected to be strongly associated with, such as measures of its established correlates. Discriminant validity refers to the measure of the construct being distinct from other measures that it is related with but theoretically distinct from. It is evidenced by the measure being not highly correlated with measures of related variables. In other words, it is a demonstration of the measure's uniqueness. In the current study, convergent and discriminate validity of the CQ-PERI-MS was examined with respect to its relationship with measures of anxiety, depression, and neuroticism as these are documented to be significant correlates of premenstrual and menstrual complaints [17,30-32] while being characteristics that are theoretically distinct from premenstrual and menstrual complaints. We hypothesized that perimenstrual symptoms as measured by the CQ- 
PERI-MS were positively and significantly related to anxiety, depression, and neuroticism but the correlations would only be moderate in strength.

\section{Other Measures}

\section{Chinese Questionnaire of Perimenstrual Symptoms (CQ-PERI-MS)}

The 20 locally derived items elicited from the focus group interviews were added to MDQ to form the 67-item CQ-PERI-MS. Participants were asked to rate the severity of each symptom on a 6-point scale, based on experiences in the previous six months. The severity of each symptom was rated separately for premenstrual, menstrual, and intermenstrual phases. Higher scores represent greater severity of symptoms.

\section{$\underline{\text { Hospital Anxiety and Depression Scale [HADS; 33,34] }}$}

Anxiety and depression were assessed by the validated Chinese version of the Hospital Anxiety and Depression Scale [HADS; 33,34]. The HADS is a 14-item selfreport instrument with two separate subscales for assessing depressive and anxiety symptoms among medical patients and the general population. Participants were asked to rate the degree to which they agree to each of the 14 statements that describe the symptoms using a four-point scale. Higher scores indicated higher levels of anxiety or depression.

\section{Neuroticism Subscale of the Eysenck Personality Inventory [N-EPI; 35$]$}

Neuroticism was measured by the Neuroticism subscale of the Eysenck Personality Inventory [35]. This 12-item subscale measures an individual's neurotic personality traits. Participants were asked to rate whether or not each statement was true of their feelings and behaviors. The total number of "yes" items represented the participant's Neuroticism score. Higher scores indicated higher levels of neuroticism.

\section{Results}

The demographic and menstrual characteristics of the sample in Study 1 are 
presented in Table 1. The mean age of the sample was 20.18 [SD=7.17]. Most of the participants were single (92.4\%).

\section{Insert Table 1 about here}

Exploratory factor analysis (EFA) using principal axis factoring and varimax rotation was performed on the initial 67-item CQ-PERI-MS, separately for the premenstrual and the menstrual phases. Only items with factor loadings greater than .40 on the same factor for both phases were included into a factor to make the resultant factor structure applicable to both the premenstrual and menstrual phases.

Since the original MDQ had eight factors, an eight-factor solution was fitted into exploratory factor analysis (EFA) using principal axis factoring and varimax rotation but the structure was not interpretable. Scree plot and eigenvalues suggested an optimal number of four factors but two to five factor solutions were also fitted to identify the most interpretable structure. Factor solutions showed that the four-factor structure was the most interpretable and parsimonious structure. Thirty-two items with factor loadings greater than .40 for both phases yielded the final CQ-PERI-MS.

Table 2 shows the factor structure, composition, and loadings of the final CQPERI-MS. Factor 1 was labeled Dysphoria, and consisted of 15 items of negative emotional states. Factor 2 was labeled Somatic Distress, and included nine items of physical symptoms. Factor 3 was labeled Cognitive Problems, and consisted of three items describing states of confusion and a lack of clarity of mind. The final factor was labeled Arousal, and included five items on various positive states such as excitement. Twelve of the twenty locally derived items loaded saliently on one of the four factors of the CQ-PERI-MS. All of them loaded either on the Dysphoria or the Somatic Distress factors, showing the prominence of these symptoms among Chinese women. The four factors together accounted for a total of $60.3 \%$ and $57.6 \%$ of variances in premenstrual and menstrual phases, respectively.

Factor analysis of scores in the intermenstrual phase did not yield any interpretable structure using the eight factor solution or the two- to five-factor solutions. An examination of the scree plot and eigenvalues suggested a fourteen factor solution which did not make any conceptual sense. This showed that the symptom ratings in the intermenstrual phase did not fall into any meaningful pattern, thereby contributing to the construct validity of the final 32-item CPDQ.

Insert Table 2 about here 
Reliability, in terms of internal consistency, was high for the four factors in both the premenstrual and the menstrual phases. Cronbach's alphas for the Dysphoria, Somatic Distress, Cognitive Problems, and Arousal subscales were .96, .92, .89, and .80 respectively in the premenstrual phase, and $.96, .78, .89$, and .78 respectively in the menstrual phase.

MANOVAs revealed that those who were menstruating at the time of filling out the questionnaires did not differ significantly on reported premenstrual symptoms (Wilks $\Lambda=.98, F_{4}=0.80, P=.384$ ) or menstrual symptoms (Wilks $\Lambda=.97, F_{4}=$ $2.01, \mathrm{P}=.094)$ from those who were not. As a result, the two groups were collapsed in subsequent analyses.

Mean factor scores for premenstrual, menstrual, and intermenstrual phases were presented in Table 3. Subsequent paired t-tests showed evidence of premenstrual and menstrual distress, as there were significant increases in symptom severity in Dysphoria $\left(t_{534}=14.36, p<.001\right)$, Somatic Distress $\left(t_{534}=14.62, p<.001\right)$, and Cognitive Problems $\left(t_{532}=6.07, p<.001\right)$, but decreases in Arousal $\left(t_{530}=-7.78, p\right.$ $<.001$ ) between the premenstrual and intermenstrual phases and between the menstrual and intermenstrual phases (Dysphoria: $t_{534}=21.73, p<.001$; Somatic Distress: $t_{534}=26.86, p<.001$; Cognitive Problems: $t_{532}=9.41, p<.001$; Arousal: $\left.t_{530}=-10.62, p<.001\right)$.

\section{Insert Table 3 about here}

To examine the convergent and discriminant validity of the CQ-PERI-MS, Pearson $r$ correlation analyses with Bonferroni correction were conducted to determine associations among premenstrual symptoms, menstrual symptoms, anxiety, depression, and neuroticism. Results are shown in Table 4. The correlation coefficients were in the expected pattern, with premenstrual and menstrual Dysphoria, Somatic Distress, and Cognitive Problems being significantly but not strongly correlated with anxiety, depression, and neuroticism, providing evidence of convergent and discriminant validity.

\section{Insert Table 4 about here}

\section{STUDY 2}

\section{Methods}




\section{Participants and Procedures}

The final CQ-PERI_MS was administered to a separate sample of Chinese females in Study 2 to confirm the factor structure derived in Study 1, and to examine the pattern of perimenstrual symptoms. Inclusion and exclusion criteria were the same as in Study 1. As in Study 1, confidentiality was assured and informed consent was sought prior to participation. Five hundred questionnaires were administered to a community sample of Hong Kong Chinese women. A total of 339 valid questionnaires were received, yielding a response rate of $67.8 \%$.

\section{Results}

The mean age of the participants in Study 2 was older than that in Study 1 $(37.28 \pm 9.39$ vs $20.18 \pm 7.17, p<.001)$ with a range of 18 to 50. Participants in Study 2 were also more diverse in terms of marital status. The majority of them were married (69\%) with $26.8 \%$ being single, and $4.1 \%$ being separated, divorced or widowed. The majority of the women $(83 \%)$ were engaged in paid employment. Their menstrual cycle characteristics were similar to those of participants in Study 1. The mean length of the menstrual cycle was 29.14 days $(\underline{S D}=2.83)$ and the mean number of days of menstrual flow was 5.63 days $(\underline{S D}=1.38)$. The majority of the participants $(72 \%)$ were not taking contraceptive pills.

Confirmatory factor analyses (CFA) were performed using the EQS Programme to determine whether the data fitted the four-factor structure identified. For the premenstrual phase, the model fit was marginally satisfactory as shown by various goodness of fit indices $(\mathrm{NFI}=.74, \mathrm{NNFI}=.76, \mathrm{CFI}=.78, \mathrm{IFI}=.78$, standardized RMR $=.08$, RMSEA $=.11$ ). As modifications suggested by the LM test did not improve the model, the original model was retained. Similarly, the model fit for the menstrual phase was also marginally satisfactory $(\mathrm{NFI}=.74, \mathrm{NNFI}=.78, \mathrm{CFI}$ $=.80, \mathrm{IFI}=.80$, standardized RMR $=.07, \mathrm{RMSEA}=.10)$. Reliability in terms of internal consistencies of factors were good, with the Cronbach's alphas for the Dysphoria, Somatic Distress, Cognitive Problems and Arousal subscales being $.96, .89, .77$, and .75 respectively for the premenstrual phase and $.96, .89, .79$, and .77 respectively for the menstrual phase.

The relationship between age and CQ-PERI-MS subscale scores were examined. Independent t-tests with Bonferroni correction were conducted. 
Participants aged 30 or above were not significantly different from those below 30 on any of the four premenstrual subscale scores. However, participants aged 30 or above had significantly higher menstrual Dysphoria scores (mean $=0.60, \mathrm{SD}=0.64$ ) compared to those below 30 (mean $=0.31, \mathrm{SD}=0.47, p=.000$ ). No significant differences were found for the other subscales.

The percentage of participants reporting premenstrual and menstrual symptoms is shown in Table 5. A total of $18.6 \%$ reported ten or more premenstrual symptoms while $34.2 \%$ reported ten or more menstrual symptoms. In general, menstrual symptoms were more frequently reported than premenstrual ones. Table 6 shows the percentage of participants reporting each of the 32 premenstrual and menstrual symptoms. There were both overlaps and differences in the most frequently reported symptoms in the two phases. The most frequently reported premenstrual symptom was irritability (39.9\%), followed by fatigue $(36.4 \%)$ and stomachache (36.2\%) while the most commonly reported menstrual symptom was fatigue $(62.0 \%)$, followed by abdominal cramps (60.6\%) and irritability (54.4\%).

\begin{tabular}{|l|}
\hline Insert Table 5 about here \\
\hline Insert Table 6 about here
\end{tabular}

Mean factor scores for the premenstrual, menstrual, and intermenstrual phases are shown in Table 7. Repeated Measures ANOVAs comparing subscale scores across the three phases were conducted. Results demonstrated significant overall differences in Dysphoria $\left(F_{2,562}=101.14, p<0.001\right)$, Somatic Distress $\left(F_{2,574}=181.00, p<.001\right)$, Cognitive Problems $\left(F_{2,558}=27.45, p<0.001\right)$, and Arousal $\left(F_{2,540}=27.52, p<0.001\right)$ across the phases. Planned post hoc comparisons showed evidence of premenstrual and menstrual distress, as well as menstrual exacerbation of premenstrual distress. There were significant increases in Dysphoria $\left(t_{282}=8.93, p<0.001 ; t_{281}=12.59, p<\right.$ $0.001)$, Somatic Distress $\left(t_{287}=10.19, p<0.001 ; t_{287}=16.18, p<0.001\right)$, and Cognitive Problems $\left(t_{280}=3.75, p<0.001 ; t_{279}=6.11, p<0.001\right)$ in the premenstrual and the menstrual phases compared to the intermenstrual phase. Moreover, Dysphoria $\left(t_{290}=5.25, p<0.001\right)$, Somatic Distress $\left(t_{297}=11.07, p<0.001\right)$, and Cognitive Problems $\left(t_{281}=4.93, p<0.001\right)$ were all significantly higher in the menstrual phase than in the premenstrual phase. Arousal decreased in both the premenstrual $\left(t_{532}=-\right.$ $7.78, p<0.001)$ and menstrual $\left(t_{530}=-10.62, p<0.001\right)$ phases, and was higher in the premenstrual phase than in the menstrual phase $\left(t_{530}=-6.03, p<0.001\right)$. 


\section{Insert Table 7 about here}

\section{Discussion}

The present study represents an effort to develop a locally valid and reliable instrument for measuring perimenstrual symptoms among Chinese women, and to use this instrument to better understand their perimentstrual distress. Previous studies in the area were seriously limited by the fact that instruments used were simply translated from the West which might not validly measure perimenstrual symptoms among Chinese women. The construction of the Chinese Questionnaire of Perimenstrual Symptoms (CQ-PERI-MS), based on a widely used Western instrument supplemented by local items, took on a culturally sensitive approach that aimed to overcome the problem. The CQ-PERI-MS was found to have good reliability in both the premenstrual and menstrual phases in Study 1 as well as in Study 2. Its factorial validity was supported by the interpretable four-factor structure derived in Study 1 which was confirmed in Study 2. Chinese women's perimenstrual experiences were characterized by four dimensions. Arousal represented more positive experiences while Dysphoria, Somatic Distress, and Cognitive Problems represented more negative experiences. Although the eight-factor structure of the MDQ [24] failed to be replicated among Chinese women, the content of the Dysphoria, Cognitive Problems, and Arousal subscales of the CQ-PERI-MS were highly similar to Moos' Negative Affect, Concentration, and Arousal subscales respectively. The Somatic Distress subscale of the CQ-PERI-MS included items that were distributed among Moos' Pain, Autonomic Reactions, and Behavioral Change subscales. This interesting finding suggests that Chinese women's perimenstrual distress is probably less elaborated than that of their Western counterparts. Various forms of physical discomfort appeared to be interfused in the experience of Chinese women. Women in Moo's study [24] experienced three distinct dimensions of somatic and behavioral symptoms (pain, autonomic reactions, and behavioral change) but somatic and behavioral symptoms of Chinese women in our study constituted one single factor which we labeled as Somatic Distress. This factor included a mixture of pain, autonomic and general physical symptoms as well as behavioral change. Different categories of somatic distress are experienced as a single entity by Chinese women. It is not entirely clear why this is so but one possibility is that traditional Chinese beliefs on health and wellbeing might have mediated Chinese women's perimenstrual experiences. As 
suggested by different researchers, perimenstrual experiences are shaped by cultural beliefs [8, 18]. The fact that different categories of somatic symptoms were experienced holistically as a single dimension of somatic distress among Chinese women appears to be very much in line with Traditional Chinese Medicine's conceptualization of health and illness. Instead of adopting an anatomical model of the body as in Western medicine, Traditional Chinese Medicine adopts a functional model, with the body conceptualized as an integrated whole and the function of all organs being interconnected, resulting in problem in one organ affecting the functioning of all other organs [36,37]. Health is conceptualized as the result of harmonious balance among interconnected systems of the body, and disease as a result of disruptive imbalance. According to Traditional Chinese Medicine, premenstrual symptoms are caused by Liver qi stagnation (or stagnated flow of vital energy of Liver) that affects multiple systems and organs [36,38]. Chinese women's perimenstrual experiences might be shaped by Traditional Chinese perspectives, with somatic symptoms relating to different body systems being experienced holistically and in an integrated manner as a single entity of physical discomfort. The item "take naps, stay in bed" also loaded saliently on the Somatic Distress factor, suggesting that the physical symptoms are associated with behavior change in the form of taking naps or staying in bed. The factor structure of the MDQ or the modified MDQ (which included only the six symptom subscales and excluded the Arousal subscale and the Control subscale) has been the subject matter of a number of studies in the literature. There is great consistency across studies regarding the Negative Affect and Concentration/Cognitive Problems factors. Most studies were able to replicate Moos' study and indicated these two symptom groups as two distinct factors [39-41]. Our study adds to this accumulating body of evidence that negative affect and cognitive problems constitute two salient groups of perimenstrual problems and confirmed the findings of previous studies [24, 39-42] that negative affect and cognitive problems constitute two core and independent dimensions of perimenstrual symptoms. The main inconsistency appears to be with somatic symptoms. While Moos [24] and Clare [39] and more recently, Ross et al. [40], all identified three distinct groups of somatic symptoms (pain, water retention, and autonomic reactions), Siegel et al [42] and van der Ploeg [41] only identified two distinct somatic factors, labeled as Physical Discomfort and Water Retention in Siegel et al's study [42] and as Pain and Water Retention in van der Ploeg's study [41]among Dutch women. In our study of Chinese 
women, somatic distress emerged as a single factor. This points to the possibility of cultural variation in perimenstrual symptoms, particularly with respect to somatic problems. It also suggested that the MDQ, used without suitable modifications and validation, may not be appropriate for women of all cultures.

Evidence for the construct validity of the CQ-PERI-MS was found in both Study 1 and Study 2 of the present study. Dysphoria, Somatic Distress, and Cognitive Problems subscale scores were significantly higher in the premenstrual and menstrual phases than in the intermenstrual phase, and Arousal subscale score was significantly lower in the premenstrual and menstrual phases than in the intermenstrual phase. This showed that the CQ-PERI-MS measures dimensions of experience that vary across different phases of the menstrual cycle. As no interpretable factor structure could be identified for the intermenstrual phase, it provided further evidence that the CPDQ measures premenstrual and menstrual distress that is structurally different from that in the intermenstrual phase.

Relationships between CQ-PERI-MS subscale scores and measures of anxiety, depression, and neuroticism provided support for the convergent and discriminant validity of the CQ-PERI-MS. All the correlation coefficients between the Dysphoria, Somatic Distress, and Cognitive Problems subscales on one hand and anxiety, depression, and neuroticism on the other were statically significant but in the range of 0.09 to 0.30 which correspond to a small to medium effect size [43]. This significant though modest strength of relationship between CQ-PERI-MS subscales and related constructs as hypothesized reflects that the CQ-PERI-MS is measuring symptom clusters that are unique and distinct from anxiety, depression and neuroticism but at the same time significantly related to these factors.

The fact that the factor structure and reliability of the CQ-PERI-MS were consistent across the two separate samples which were quite different in age and marital status supports that it is a valid and reliable measure of perimenstrual symptoms among Hong Kong women in general.

The present study demonstrated the usefulness of integrating locally derived items with those drawn from existing research instruments $[44,45]$. This approach to research in non-Western populations is superior to the straightforward deletion of useless items from a standard Western questionnaire [46]. Many of the locally derived items, for example, worrisome, paleness, body dissatisfaction, weakness, and faultfinding had high endorsement frequency and loaded saliently on the four factors. This 
showed that the local items are prominent and constitute an indispensable part of the Chinese perimenstrual experience. Failure to include these items would lead to a falsely low prevalence of perimenstrual distress among Chinese women, as well as limit our understanding of the complete spectrum of perimenstrual symptoms among these women.

It is noteworthy that many of these locally derived items are culturally sensitive but not culturally specific. Indeed, some of these items do overlap with items on measures of premenstrual symptoms in the West. For example, the 95-item Western derived Premenstrual Assessment Form [PAF; 47] also consists of an item on feelings of weakness. The locally derived items "worrisome" and "body dissatisfaction" are also similar to though not exactly the same as the items "pessimistic outlook" and "dissatisfaction with appearance" on the PAF. These symptoms are culturally sensitive in describing Chinese women's perimenstrual experiences but are by no means specific to Chinese women. Some locally derived items such as "paleness" are quite culturally specific and not found in any of the existing Western-derived instruments.

Even though some of the locally elicited symptoms have some degree of overlap with items on the PAF, the significance of these symptoms and the CQ-PERIMS should not be seen as being discounted. This is because the PAF is very lengthy and is thus not a very practical research instrument. The CQ-PERI-MS is a much more concise representation of the range of perimenstrual symptoms experienced by Chinese women. Its brevity is important in facilitating further perimenstrual research among Chinese women. Another unique significance of the CQ-PERI-MS is that while the PAF is only a measure of premenstrual symptoms, the CQ-PERI-MS is a measure of both premenstrual and menstrual symptoms. It will serve as an invaluable tool in future studies of perimenstrual distress.

Although some items on the CQ-PERI-MS such as paleness seem to be very culturally specific, this does not preclude the possibility that women in other cultures also experience these symptoms. It is plausible that these were not included in instruments developed in the West and hence not assessed among Western women. Future studies should aim at examining the existence of these symptoms in nonChinese women.

Using the CQ-PERI-MS, the prevalence of perimenstrual distress among Chinese women was examined. Nearly one-fifth and more than one-third of our 
sample of Chinese women experienced ten or more premenstrual and menstrual symptoms respectively. This is comparable to the reported prevalence of perimenstrual symptoms in Western studies [48]. It is likely that the $2.9 \%$ of women who experienced more than 20 premenstrual symptoms and the $6.2 \%$ who experienced more than 20 menstrual symptoms on the in our study actually suffered from severe forms of perimenstrual distress and would require treatment. This rough estimate is similar to rates found in other studies [48-50].

The figures dispel the myth that Chinese women do not suffer from perimenstrual distress. It also adds to the accumulating body of evidence that perimenstrual distress is a cross-cultural phenomenon [17] rather than a Western "culture-bound syndrome". There are increasing reports of premenstrual complaints from urbanized regions of developing countries such as India [9,10], Africa [51] and China [11-14], though the reported rates are generally lower than those identified in the present study. The use of a culturally valid instrument in the present study might have contributed to a more accurate estimation of the prevalence of premenstrual symptoms among Chinese women. It also provides much needed information on the prevalence of menstrual symptoms among Chinese women.

Contrary to the widely held notion that Chinese people are not as psychologically-minded as their Western counterparts, and tend to "somatize" their distress, our findings of the high endorsement frequency of emotional symptoms and the fact that Dysphoria emerged as the largest factor might seem surprising. These supported the view that somatization in Chinese populations has been over-stated [52]. It is also worth noting that diverse emotional states including irritability, anger, depressed mood, and anxiety were endorsed by the participants. Irritability was more commonly reported premenstrual symptom than depressed mood. The same observation was documented in a number of studies among Western women [1,53,54] as well as among Indian women [10], Chinese women in Hong Kong [55], and African women [51]. In our study, we further found that irritability constitute a core emotional symptom in the menstrual phase. Taken together, these findings highlight the need to recognize irritability as a core element of perimenstrual emotional distress in clinical practice, and the need to include this item in any research instrument developed for measuring perimenstrual symptoms.

Another interesting finding is the prominence of fatigue in both the premenstrual $(36.4 \%)$ and menstrual $(62.0 \%)$ phases. These rates were higher than 
those of Indian women [10], African women [51], and women in the USA [56], but were in keeping with a previous study of premenstrual symptoms among Chinese women in Hong Kong [55] and a local community health survey which revealed that $71 \%$ of women reported frequent fatigue [57]. Our study showed that an appreciable portion of women reported exacerbation of fatigue before or during menstruation. The salience of this symptom among Chinese women should be given due attention both in research and in clinical practice.

Menstrual distress was shown to be more common than premenstrual ones. This affirmed that menstrual distress should not be left out in the study of perimenstrual distress, as is the case in the current literature. It also appeared that the relationship between menstrual symptoms and the psychological variables of anxiety, depression, and neuroticism was much stronger than that between premenstrual symptoms and these variables. Taken together, it highlights the significance of menstrual distress as an issue that certainly deserves greater research and clinical attention.

Given that the CQ-PERI-MS seems to be culturally sensitive but not necessarily culturally specific to Chinese women, future studies could also test its validity among Western women. Given that experiences change with time and changing social forces, it is in all likelihood that the additional perimenstrual symptoms identified in the present study and included in the CQ-PERI-MS represent new forms of distress brought about by modernization rather than being due to cultural difference per se. Studies aiming to examine cultural myths and attitudes towards menstruation, especially as they relate to perception, recognition, and reporting of symptoms, should also be encouraged

Several limitations of the present study need to be highlighted. First, convenience sampling limited the generalizability of the findings, and the study should be taken as exploratory. Second, Hong Kong women are not representative of the whole Chinese female population. Caution should be exercised in extrapolating results to Chinese females in other social and cultural settings. Third, data were collected from retrospective self-reports. There was no clinical verification of the perimenstrual symptoms. The retrospective nature of the self-report also means that the cyclicity of symptom variation, as reported, may be amplified, though it was admitted that retrospective reports were reasonably accurate among women who experienced moderate or severe symptoms [48]. There is also evidence that the factor 
structure of perimenstrual distress was stable irrespective of whether the symptoms were assessed retrospectively or prospectively [40]. We can, therefore, be reasonably confident that despite the retrospective nature of our study, our data adequately reflects both the structure and the rates of perimenstrual symptoms among Chinese women. Future studies should, however, confirm our findings through the use of prospective assessments, supplemented by clinician's verification of symptom severity and functional impairment.

Notwithstanding these limitations, our study demonstrated the validity and reliability of the CQ-PERI-MS in assessing premenstrual and menstrual symptoms among Chinese women, and the significance of perimenstrual symptoms among this group of women who were once believed to be minimally affected by this problem. It also contributes to our understating of the structure of perimenstrual symptoms crossculturally.

\section{$\underline{\text { References }}$}

1. Angst J, Sellaro R, Stolar M, Merikangas KR, Endicott J. The epidemiology of perimenstrual psychological symptoms. Acta Psychiatr Scand 2001;104:110116.

2. Santer M, Wyke S, Warner P. What aspects of periods are most bothersome for women reporting heavy menstrual bleeding? Community survey and qualitative study. BMC Women's Health 2007;7:8.

3. White PA, Wildman BG. Factors related to medical help-seeking in women with menstrual discomfort. Behav Res Ther 1986;24:471-474.

4. Bancroft J. The premenstrual syndrome - a reappraisal of the concept and the evidence. Psychol Med 1993;(Suppl. 24): 1-47.

5. Bancroft J. The menstrual cycle and the well being of women. Soc Sci Med 1995;41:785-791.

6. Johnson T. Premenstrual syndrome as a Western culture-specific disorder. Cult Med Psychiatry 1987; 11:337-56.

7. Chrisler JC. PMS as a culture-bound syndrome In: Chrisler JC, Golden C \& Rozee PD, editors. Lectures on psychology of women $\left(4^{\text {th }}\right.$ ed.). New York: McGraw-Hill; 2008. 
8. Chrisler JC, Caplan P. The strange case of Dr. Jekyll and Ms. Hyde: How PMS became a cultural phenomenon and a psychiatric disorder. Annu Rev Sex Res 2002; 13: 274-306.

9. Chandra P, Chaturvedi S. Cultural variations of premenstrual experience. Int J Soc Psychiatry 1989; 35:343-9.

10. Chaturvedi S, Chandra P, Gururaj G, Beena M, Pandian R. Prevalence of premenstrual symptoms and syndromes: preliminary observations. Nimhans $\mathbf{J}$ 1994; 12:9-14.

11. Rossignol A, Zhang J, Chen Y, Zheng X. Tea and premenstrual syndrome in the People's Republic of China. Am J Public Health 1989; 79:67-9.

12. $\mathrm{Xu} \mathrm{S}$, Zhu P. An exploration of premenstrual syndrome and women's psychology. Chin J of Clin Psychol 1997; 5:246-7 (in Chinese).

13. Yu M, Zhu X, Li J, Oakley D, Reame N. Perimenstrual symptoms among Chinese women in an urban area of China. Health Care for Women Int 1996; $17: 161-72$.

14. Yuan H, Zhou P, Wan X, Zhang L, Cai J and Zhai S. Women's coping mechanisms for premenstrual syndrome. Chin J Nerv Ment Dis 1997; 23:97-8.

15. Takeda T, Tasaka K, Sakata M, Murata Y. Prevalence of premenstrual syndrome and premenstrual dysphoric disorder in Japanese women. Arch Womens Ment Health 2006;9:209-212

16. Chayachinda C, Rattanachaiyanont M, Phattharayuttawat S, Kooptiwoot S. Premenstrual Syndrome in Thai nurses. J Psychosom Obstet Gynecol 2008;29:199-205.

17. Adewuya AO, Loto OM, Adewumi TA. Premenstrual dysphoric disorder amongst Nigerian university students: prevalence, comorbid conditions, and correlated. Arch Women's Mental Health 2008;11:13-18.

18. Sveindottir H. Prospective assessment of menstrual and premenstrual experiences of Icelandic women. Health Care for Women Int 1998; 19:71-82.

19. Dan AJ, Monagle L. Sociocultural influences on women's experiences of perimenstrual symptoms In: Gold JH, Severino SK, editors. Premenstrual Dysphorias: Myths and Realities. Washington DC: American Psychiatric Press; 1994. 
20. World Health Organization. A cross-cultural study of menstruation: Implications for contraceptive development and use. Study of Fam Planning 1981; 12:17-27.

21. World Health Organization. Women's bleeding patterns: Ability to recall and predict menstrual events. Study of Fam Planning 1981; 12:17-27.

22. Chang A, Holroyd E, Chau J. Premenstrual syndrome in employed Chinese women in Hong Kong. Health Care for Women Int 1995; 16:551-61.

23. Yu M, Zhu X, Li J, Oakley D, Reame N. Perimenstrual symptoms among Chinese women in an urban area of China. Health Care for Women Int 1996; 17:161-72.

24. Moos R. The development of a menstrual distress questionnaire. Psychosom Med 1968; 30:853-67.

25. Kleinman, AM. Anthropology and psychiatry: the role of culture in crosscultural research on illness. Br J Psychiatry 1987; 151:447-454.

26. Glaser, B. and Strauss, A. (1967). The discovery of grounded theory: Strategies for qualitative research. New York: Aldine Publishing Company.

27. Guest G, Bunce A, Johnson L. How many interviews are enough? An experiment with data saturation and variability. Field Methods 2006;18:59-82.

28. Campbell DT, Fiske DW. Convergent and discriminant validation by the multitrait-multimethod matrix. Psychological Bulletin 1959;56:81-105.

29. Kaplan RM, Saccuzzo DP. Psychological testing: Principles, applications, and issues $\left(6^{\text {th }}\right.$ ed). 2005. Wadsworth:CA.

30. Taylor JW. Psychological factors in the aetiology of premenstrual symptoms. Aust NZ J Psychiatry 1979;13:35-41.

31. Veeninga AT, DeRuiter C, Kraaimaat FW. The relationship between late luteal phase dysphoric disorder and anxiety disorders. $\underline{\mathrm{J} \text { Anxiety Disorders }}$ 1994;8:207-215.

32. Watts S, Dennerstein L, Del Horne DJ. The premenstrual syndrome: a psychological evaluation. J Affective Disorders 1980;2:257-266.

33. Zigmond A, Snaith R. The Hospital Anxiety and Depression Scale. Acta Psychiatr Scand 1983; 67:361-70.

34. Leung C, Ho S, Kao C, Hung C, Chen C. Evaluation of the Chinese version of the Hospital Anxiety and Depression Scale: A cross-cultural perspective. Int J Psychosomatics 1992; 40:29-34. 
35. Eysenck HJ, Eysenck SBJ. Personality and individual differences: A natural science approach. New York: Plenum Press; 1985.

36. Chou PBY, Morse CA. Understanding premenstrual syndrome from a Chinese medicine perspective. J Alternate Complementary Med 2005;11:355-361.

37. Fabrega $\mathrm{H}$. The concept of somatization as a cultural and historical product of Western medicine. Psychosom Med 1990;52:653-672.

38. Chou PB, Morse CA, Xu H. A controlled trial of Chinese herbal medicine for premenstrual syndrome. J Psychosom Obstet Gynecol 2008;29:185-192.

39. Clare AW. Psychiatric and social aspects of premenstrual complaint. Psychol Med 1983;4 (suppl):1-58.

40. Ross C, Coleman G, Stojanovska C. Factor structure of the modified Moos Menstrual Distress Questionnaire: assessment of prospectively reported follicular, menstrual and premenstrual symptomatology. J Psychosom Obstet Gynecol 2003;24:163-174.

41. van der Ploeg H. The factor structure of the Menstrual Distress QuestionnaireDutch. Psychol Rep 1990; 66:707-14.

42. Siegel JP, Myers BJ, Dineen MK. Premenstrual tension syndrome symptom clusters: Statistical evaluation of the subsyndromes. J Reprod Med 1987;32:395-399.

43. Cohen J. Statistical Power Analysis for the Behavioral Sciences ( $2^{\text {nd }}$ ed). Hillsdale, N. J.: Lawrence Erlbaum Associates 1988.

44. Cheung F, Leung K, Fan R, Song W, Zhang J, Zhang J. Development of the Chinese Personality Assessment Inventory. J Cross Cult Psychol 1996; 27:181-99.

45. Kleinman A. Rethinking psychiatry: From cultural categories to personal experience. New York: Free Press; 1988.

46. Chaturvedi S, Chandra P. Sociocultural aspects of menstrual attitudes and premenstrual experiences in India. Soc Sci Med 1991; 32:349-51.

47. Halbreich U, Endicott J, Schach S, Nee J. The diversity of premenstrual changes as reflected in the premenstrual assessment form. Acta Psychiatr Scand 1982;62:177-190.

48. Logue C, Moos R. Perimenstrual symptoms: Prevalence and risk factors. Psychosom Med 1986; 48:388-415. 
49. Rivera-Tovar A, Frank E. Late luteal phase dysphoric disorder in young women. Am J Psychiatry 1990; 147:1634-6.

50. Yonkers KA, Davis, LL. Premenstrual dysphoric disorder In: Sadock BJ, Sadock VA, editors. Kaplan and Sadock's Comprehensive Textbook of Psychiatry, 7th ed. Philadelphia: Williams \& Wilkins; 2000.

51. McMaster J, Cormie K, Pitts M. Menstrual and premenstrual experiences of women in a developing country. Health Care Women Int 1997;18:533-541.

52. Kirmayer L, Young A. Culture and somatization: Clinical, epidemiological, and ethnographic perspectives. Psychosom Med 1998; 60:420-30.

53. Hartlage SA, Arduino KE. Toward the content validity of premenstrual dysphoric disorder: Do anger and irritability more than depressed mood represent treatment-seekers' experience?

54. Landen M, Eriksson E. How does premenstrual dysphoric disorder relate to depression and anxiety disorders? Depress Anx 2003;17:122-129.

55. Lee AM, Wei R, Chung KF, Hui KT, Ip SK, Leung HL, Liu HL, Lui SY, Ng YH, Wong MF, Wong TC. Premenstrual Syndrome among Chinese female undergraduates: Relationship with stress and mental health. Hong Kong Journal of Gynaecology, Obstetrics and Midwifery 2005;5:10-21.

56. Woods NF, Dery GK, Most A.Recollections of menarche, current menstrual attitudes, and perimenstrual symptoms. Psychosom Med 1982;44:285-293.

57. Tang C, Lee AM, Tang T, Cheung FM \& Chan C. Role occupancy, role quality, and psychological distress in Chinese women. Women \& Health 2002;36:49-66. 
Table 1. Demographic and Menstrual Characteristics of the Sample in Study 1

\begin{tabular}{l|l}
\hline & \multicolumn{1}{|c}{$n=538$} \\
\hline Age & $20.18[\mathrm{SD}=7.17]$ \\
$14-18$ & $211(39.6)$ \\
$19-25$ & $262(49.2)$ \\
$26-35$ & $28(5.2)$ \\
$36-45$ & $18(3.4)$ \\
46 and above & $14(2.6)$ \\
Marital status & \\
Single & $489(92.4)$ \\
Married & $39(7.4)$ \\
Separated/Divorced & $1(0.2)$ \\
Menstrual Characteristics & \\
Age of menarche & $12.27[\mathrm{SD}=1.31]$ \\
Length of menstrual cycle & $30.58[\mathrm{SD}=5.54)$ \\
Length of menstrual flow & $5.73[\mathrm{SD}=1.19]$ \\
Taking oral contraceptive & \\
No & $527(99.4)$ \\
Yes & $3(0.6)$
\end{tabular}

Note: Values are given as $\mathrm{n}(\%)$ or mean [SD].

Percentages are expressed as valid percentages. 
Table 2. Factor Structure of the CQ-PERI-MS for Study $1(n=538)$

\begin{tabular}{|c|c|c|c|}
\hline & & \multicolumn{2}{|c|}{ Salient Factor Loadings } \\
\hline & & Premenstrual & Menstrual \\
\hline \multirow[t]{16}{*}{ Factor 1: } & Dysphoria & $25.0 \%$ & $27.0 \%$ \\
\hline & Loneliness & .56 & .61 \\
\hline & Anxiety & .50 & .59 \\
\hline & Irritability & .72 & .75 \\
\hline & Mood swings & .78 & .81 \\
\hline & Depressed mood & .78 & .74 \\
\hline & Tension & .57 & .64 \\
\hline & Hypersomnia (L) & .50 & .54 \\
\hline & Impulsive (L) & .63 & .71 \\
\hline & Anger (L) & .85 & .83 \\
\hline & Fault-finding/Unpleasant (L) & .79 & .82 \\
\hline & Impatience $(\mathrm{L})$ & .81 & .82 \\
\hline & Worrisome (L) & .75 & .75 \\
\hline & Feelings of loss of control (L) & .70 & .72 \\
\hline & Body dissatisfaction (L) & .43 & .55 \\
\hline & Easy to lose temper (L) & .80 & .81 \\
\hline \multirow[t]{10}{*}{ Factor 2: } & Somatic Distress & $15.8 \%$ & $17.6 \%$ \\
\hline & Take naps, stay in bed & .57 & .62 \\
\hline & Abdominal cramps & .75 & .74 \\
\hline & Dizziness, faintness & .58 & .65 \\
\hline & Backache & .49 & .43 \\
\hline & Fatigue & .64 & .62 \\
\hline & Nausea, vomiting & .50 & .59 \\
\hline & Stomachache & .77 & .75 \\
\hline & Weakness (L) & .55 & .64 \\
\hline & Paleness (L) & .44 & .64 \\
\hline \multirow[t]{4}{*}{ Factor 3: } & Cognitive Problems & $11.8 \%$ & $17.6 \%$ \\
\hline & Confusion & .57 & .50 \\
\hline & Lowered judgment & .72 & .59 \\
\hline & Blurred vision, blindspots & .59 & .47 \\
\hline \multirow[t]{6}{*}{ Factor 4: } & Arousal & $11.8 \%$ & $7.0 \%$ \\
\hline & Excitement & .51 & .48 \\
\hline & Affectionate & .44 & .40 \\
\hline & Orderliness & .62 & .61 \\
\hline & Feelings of well-being & .89 & .91 \\
\hline & Bursts of energy, activity & .90 & .86 \\
\hline
\end{tabular}

Note: \% refer to the amount of total variance accounted for by the respective factors; (L) denotes local items that were not in the original MDQ 
Table 3. Comparison of Premenstrual versus Intermenstrual and Menstrual versus Intermenstrual CQ-PERI-MS Subscale Scores in Study $1(n=538)$. Values are given as mean $[\mathrm{SD}]$

\begin{tabular}{lllll}
\hline CQ-PERI-MS & Premenstrual & Menstrual & Intermenstrual & $P$
\end{tabular}

$\begin{array}{llllll}\text { Subscales } & (\mathrm{P}) & (\mathrm{M}) & (\mathrm{I}) & (\mathrm{P} v s \mathrm{I}) & (\mathrm{M} v s \mathrm{I})\end{array}$

$\begin{array}{llllll}\text { Dysphoria } & 1.65[.89] & 1.85[.92] & 1.29[.63] & <.001 & <.001\end{array}$

$\begin{array}{llllll}\text { Somatic Distress } & 1.54[.71] & 2.02[.88] & 1.21[.45] & <.001 & <.001\end{array}$

$\begin{array}{llllll}\text { Cognitive Problems } & 1.15[.46] & 1.24[.56] & 1.08[.35] & <.001 & <.001\end{array}$

$\begin{array}{lllllll}\text { Arousal } & 1.53[.79] & 1.41[.68] & 1.72[.90] & <.001 & <.001\end{array}$

Note: $\mathrm{P} v s$ I represent Premenstrual Changes, $\mathrm{M}$ vs I represent Menstrual Changes 
Table 4. Table showing correlations of premenstrual symptoms and menstrual symptoms, with anxiety, depression, and neuroticism.

\begin{tabular}{l|ccc}
\hline & Anxiety & Depression & Neuroticism \\
\hline Premenstrual & $.20^{* *}$ & $.10^{*}$ & $.18^{* *}$ \\
Dysphoria & $.19^{* *}$ & $.11^{* *}$ & $.14^{* *}$ \\
Somatic Distress & $.20^{* *}$ & $.11^{*}$ & $.11^{* *}$ \\
Cognitive Problems & $\mathrm{NS}$ & $\mathrm{NS}$ & $\mathrm{NS}$ \\
Arousal & $.30^{* *}$ & & $.30^{* *}$ \\
Menstrual & $.24^{* *}$ & $.14^{* *}$ & $.25^{* *}$ \\
Dysphoria & $.26^{* *}$ & $.09^{*}$ & $.18^{* *}$ \\
Somatic Distress & $\mathrm{NS}$ & $.15^{* *}$ & $.10^{*}$ \\
Cognitive Problems & NS & & \\
Arousal &
\end{tabular}

Note: $* \mathrm{p}<.05 ; * * \mathrm{p}<.01, \mathrm{NS}$ not significant 
Table 5. Percentage of participants $(n=339)$ experiencing ten or more, fifteen or more, and twenty or more premenstrual and menstrual symptoms (Study 2).

\begin{tabular}{lll}
\hline & Premenstrual & Menstrual \\
\hline 10 or more symptoms & $63(18.6)$ & $116(34.2)$ \\
15 or more symptoms & $25(7.4)$ & $60(17.7)$ \\
20 or more symptoms & $10(2.9)$ & $21(6.2)$ \\
\hline
\end{tabular}

Note: Values are given as $\mathrm{n}(\%)$. 
Table 6. Percentage of Participants Reporting Premenstrual and Menstrual Symptoms $(n=339)$

\begin{tabular}{|c|c|c|c|}
\hline & & Premenstrual & Menstrual \\
\hline \multirow[t]{16}{*}{ Factor 1: } & Dysphoria & & \\
\hline & Loneliness & $7.6 \%$ & $12.8 \%$ \\
\hline & Anxiety & $24.0 \%$ & $26.8 \%$ \\
\hline & Irritability & $39.9 \%$ & $54.4 \%$ \\
\hline & Mood swings & $32.5 \%$ & $47.1 \%$ \\
\hline & Depressed mood & $26.8 \%$ & $34.9 \%$ \\
\hline & Tension & $19.4 \%$ & $27.3 \%$ \\
\hline & Hypersomnia (L) & $18.5 \%$ & $37.1 \%$ \\
\hline & Impulsive (L) & $11.6 \%$ & $14.9 \%$ \\
\hline & Anger (L) & $22.5 \%$ & $32.7 \%$ \\
\hline & Fault-finding/Unpleasant (L) & $17.2 \%$ & $24.8 \%$ \\
\hline & Impatience (L) & $24.7 \%$ & $37.8 \%$ \\
\hline & Worrisome (fan nao) (L) & $23.3 \%$ & $31.6 \%$ \\
\hline & Feelings of loss of control (L) & $11.3 \%$ & $12.8 \%$ \\
\hline & Body dissatisfaction (L) & $10.9 \%$ & $14.6 \%$ \\
\hline & Easy to lose temper (L) & $31.5 \%$ & $40.7 \%$ \\
\hline \multirow[t]{10}{*}{ Factor 2: } & Somatic Distress & & \\
\hline & Take naps, stay in bed & $21.3 \%$ & $45.1 \%$ \\
\hline & Abdominal cramps & $33.6 \%$ & $60.6 \%$ \\
\hline & Dizziness, faintness & $14.7 \%$ & $25.7 \%$ \\
\hline & Backache & $25.5 \%$ & $36.0 \%$ \\
\hline & Fatigue & $36.4 \%$ & $62.0 \%$ \\
\hline & Nausea, vomiting & $8.2 \%$ & $10.8 \%$ \\
\hline & Stomachache (L) & $36.2 \%$ & $54.3 \%$ \\
\hline & Weakness (xu yue) (L) & $18.2 \%$ & $31.8 \%$ \\
\hline & Paleness (L) & $12.9 \%$ & $43.8 \%$ \\
\hline \multirow[t]{4}{*}{ Factor 3: } & Cognitive Problems & & \\
\hline & Confusion & $4.0 \%$ & $9.8 \%$ \\
\hline & Lowered judgment & $8.6 \%$ & $15.6 \%$ \\
\hline & Blurred vision, blindspots & $5.1 \%$ & $7.7 \%$ \\
\hline \multirow[t]{6}{*}{ Factor 4: } & Arousal & & \\
\hline & Excitement & $7.0 \%$ & $6.6 \%$ \\
\hline & Affectionate & $3.0 \%$ & $4.3 \%$ \\
\hline & Orderliness & $4.8 \%$ & $7.5 \%$ \\
\hline & Feelings of well-being & $7.5 \%$ & $7.9 \%$ \\
\hline & Bursts of energy, activity & $4.2 \%$ & $4.2 \%$ \\
\hline
\end{tabular}


Table 7. Comparison of CPDQ Subscale Symptom Severity Scores in the Premenstrual, Menstrual, and Intermenstrual Phases in Study $2(n=339)$ using Repeated Measures ANOVA and Planned Post Hoc Comparisons.

\begin{tabular}{|c|c|c|c|c|c|c|}
\hline \multirow[t]{2}{*}{$\overline{\text { CPDQ Subscale }}$} & \multirow{2}{*}{$\begin{array}{l}\text { Premenstrual } \\
\text { (P) }\end{array}$} & \multirow{2}{*}{$\begin{array}{l}\text { Menstrual } \\
\text { (M) }\end{array}$} & \multirow{2}{*}{$\begin{array}{l}\text { Intermenstrual } \\
\text { (I) }\end{array}$} & \multicolumn{3}{|c|}{ Planned Post Hoc Comparisons } \\
\hline & & & & $P(\mathrm{P} v s \mathrm{I})$ & $P(\mathrm{M} v s \mathrm{I})$ & $P(\mathrm{P} v s \mathrm{M})$ \\
\hline Dysphoria & $1.54[.77]$ & $1.66[.80]$ & $1.24[.50]$ & $<.001$ & $<.001$ & $<.001$ \\
\hline $\begin{array}{l}\text { Somatic } \\
\text { Distress }\end{array}$ & $1.53[.66]$ & $1.85[.80]$ & $1.25[.47]$ & $<.001$ & $<.001$ & $<.001$ \\
\hline $\begin{array}{l}\text { Cognitive } \\
\text { Problems }\end{array}$ & $1.19[.50]$ & $1.26[.61]$ & $1.11[.33]$ & $<.001$ & $<.001$ & $<.001$ \\
\hline Arousal & $1.34[.59]$ & $1.33[.60]$ & $1.21[.47)]$ & $<.001$ & $<.001$ & $<.001$ \\
\hline
\end{tabular}

Note: Values are given as mean [SD]. 